\title{
Influência da religiosidade sobre a saúde mental dos acadêmicos de medicina
}

\author{
Influence of religiosity on medical students' mental health
}

\author{
Larissa Cruvinel Leite1 (1) | larissacruvinelleite@gmail.com \\ Larissa Vitoria Dornelas ${ }^{1}$ (D) dornelaslarissav@gmail.com \\ Laura de Souza Bechara Secchin'1(D) laura.bechara@suprema.edu.br
}

\section{RESUMO}

Introdução: A saúde mental foi incluída como um dos dez principais indicadores de saúde, e estudos demonstraram sua relação com religiosidade e espiritualidade (R/E).

Objetivo: Este estudo teve como objetivo avaliar se o grau de R/E dos acadêmicos de Medicina influencia em transtornos de ansiedade e depressão no decorrer da graduação.

Método: Este trabalho foi realizado com uma amostra não probabilística, por conveniência, constituída de 298 acadêmicos. Utilizou-se, na forma de questionários, o Índice de Religiosidade de Duke em português brasileiro (P-DUREL), e, para mapeamento de depressão, ansiedade e estresse, aplicou-se a DASS-21.

Resultado: Foi demonstrado que as diferentes dimensões da religiosidade não possuem associação com os transtornos emocionais depressão, ansiedade e estresse dos estudantes.

Conclusões: É possível propor recomendações para pesquisas futuras, de modo que os resultados possam ser utilizados em estudos de metanálise. Estudos que não apresentaram relação significativa entre as variáveis não podem ser negligenciados para que sejam investigadas as dimensões positivas e negativas da associação entre $R /$ E e saúde mental.

Palavras-chave: Religião; Espiritualidade; Depressão; Ansiedade; Estudantes de Medicina.

\section{ABSTRACT}

Introduction: Mental health was included as one of the top ten health indicators and studies have shown it is related to religiosity and spirituality (R/S). Objective: The aim of the present study is to evaluate whether the degree of R/S of medical students influences anxiety and depression disorders during undergraduate school.

Method: This study was carried out with a convenience non-probabilistic sample, consisting of 298 students. The Duke Religiosity Index in Brazilian Portuguese (P-DUREL) was used in questionnaire form, whereas, to map depression, anxiety and stress, DASS-21 was applied.

Results: It was demonstrated that the different dimensions of religiosity have no association with the students' emotional disorders, anxiety and stress.

Conclusions: It is possible to propose recommendations for future research, so that the results can be used in meta-analysis studies. Studies that did not show a significant association between the variables cannot be neglected, so that the positive and negative dimensions of the association between $R / S$ and mental health can be investigated.

Keywords: Religion; Spirituality; Depression; Anxiety; Medical Students.

${ }^{1}$ Faculdade de Ciências Médicas e da Saúde de Juiz de Fora, Juiz de Fora, Minas Gerais, Brasil.

Editora-chefe: Rosiane Viana Zuza Diniz

Editora associada: Izabel Cristina Meister Martins Coelho

Recebido em 25/09/20; Aceito em 24/02/21.

Avaliado pelo processo de double blind review. 


\section{INTRODUÇÃO}

A saúde mental foi incluída como um dos dez principais indicadores de saúde ${ }^{1}$ nos objetivos nacionais publicados, em janeiro de 2000, no Healthy People 2010 pelo Department of Health and Human Services. Aspectos do bem-estar merecem atenção, tanto em nível teórico quanto clínico, principalmente por estarem associados a uma ampla gama de doenças², inclusive depressão e ansiedade - transtornos mentais comuns e debilitantes. Estudos demonstraram significativa relação entre saúde mental, religiosidade e espiritualidade $(R / E)^{3}$, principalmente, com a emergência do conceito "espiritualidade baseada em evidência"4.

Segundo Koenig et al. $^{5}$, religiosidade é o quanto o indivíduo acredita em uma religião, a pratica com um sistema organizado de crenças e insere-se em uma comunidade definida, enquanto espiritualidade é a busca pessoal sobre questões existenciais, como o sentido da vida, e suas relações com o transcendente, incluindo ou não uma atuação religiosa formal.

Artigos científicos mostram que intervenções espirituais aumentam o otimismo e reduzem o pessimismo, especialmente em casos de doença ${ }^{6}$, e que intervenções de R/E promovem maior aceitação e apoio social, com uma compreensão mais profunda da existência ${ }^{7}$. Na adolescência, a religião desempenha um papel importante no processo de socialização e na formação da personalidade, sendo um fator protetor contra o uso de drogas, o tabagismo e a atividade sexual precoce, mas seu efeito na saúde é menos conhecido ${ }^{8}$. Isso ocorre porque a maioria dos artigos que associaram variáveis religiosas a resultados positivos para saúde não incluíram jovens, e sim idosos e doentes 9 . Apesar desse contexto de poucos estudos, é sabido que estudantes universitários enfrentam muitos conflitos (educacionais, culturais e financeiros) ${ }^{10}$ e que acadêmicos de medicina sofrem mais estresse quando comparados aos dos outros cursos. Ademais, as taxas de depressão aumentam ao longo da formação, existindo relação significativa com doenças e queda do rendimento acadêmico ${ }^{9}$.

Embora existam estudos relacionando R/E e depressão, a ansiedade é amplamente ignorada ${ }^{8}$. Outro aspecto referente aos artigos é a dificuldade em utilizar os conceitos de R/E, muitas vezes vistos como sinônimos ${ }^{11}$. A diversidade de protocolos e resultados, associada à falta de padronização das pesquisas, aponta para a necessidade de mais estudos que avaliem a espiritualidade na saúde ${ }^{7}$.

Ao longo da vida, o indivíduo é exposto a situações que podem afetar negativamente seu bem-estar, e, no âmbito da saúde mental, suspeita-se que a religiosidade contribui como um fator protetor. $\mathrm{O}$ objetivo do presente estudo foi avaliar se o grau de R/E dos acadêmicos de Medicina, de uma faculdade de saúde em Juiz de Fora, influencia em transtornos de ansiedade e depressão no decorrer da graduação.

\section{MÉTODOS}

Este trabalho foi realizado com uma amostra não probabilística, por conveniência, constituída de 298 acadêmicos do curso de medicina de uma faculdade da rede privada de Juiz de Fora, em Minas Gerais, Brasil, de abril a junho de 2019. Os alunos cursavam o primeiro, segundo, sexto, sétimo, décimo primeiro e décimo segundo semestres de graduação à época da pesquisa (2019); a escolha dos períodos da graduação foi feita de forma que fosse possível fazer análises comparativas dos estudantes no início, no meio e no final do curso.

O estudo foi autorizado pelo Comitê de Ética em Pesquisa da faculdade em questão, sob o Certificado de Apresentação para Apreciação Ética (CAAE) nº 04329318.6.0000.5103, e os estudantes concordaram em participar, voluntariamente, por meio da assinatura do Termo de Consentimento Livre e Esclarecido.

Foram excluídos os dados referentes a 15 indivíduos que não satisfizeram o critério de inclusão do projeto, já que estavam fora da faixa etária de 18 a 30 anos. Além disso, outros 16 indivíduos não completaram corretamente variáveis-chave deste estudo e também foram excluídos da análise. Também se observou que dois indivíduos não preencheram suas respectivas idades, as quais foram completadas com a idade média dos alunos do período ao qual pertenciam. Ao todo, 267 indivíduos fizeram parte da análise estatística.

Os questionários foram aplicados aos alunos durante o período letivo, antes ou depois das aulas, na sala de aula. Os pesquisadores informaram os objetivos do estudo antes da entrega dos questionários, e estes foram preenchidos pelos próprios alunos.

Como instrumento de avaliação, utilizou-se, na forma de questionários, o Índice de Religiosidade de Duke em português brasileiro (Duke Religiosity Index in Brazilian Portuguese - P-DUREL), e, para mapeamento de depressão, ansiedade e estresse, aplicouse a Depression, Anxiety \& Stress Scale (DASS-21). Em conjunto foi disponibilizado um questionário sociodemográfico (sexo, idade, etnia, religião e semestre de graduação) para avaliar a contribuição de tais dados no resultado.

O P-DUREL é instrumento sucinto e de fácil aplicação, que permite a medida multidimensional da religiosidade. Ele apresenta cinco itens que mensuram as três principais dimensões do envolvimento religioso relacionadas a desfechos em saúde: religiosidade organizacional (RO) - reuniões religiosas; religiosidade não organizacional (RNO) - atividades religiosas privadas (como oração, meditação e leitura); e religiosidade intrínseca $(\mathrm{RI})$ - busca de internalização e vivência da religiosidade $\mathrm{e}^{12,13}$.

A DASS-21 é um conjunto de três subescalas, do tipo Likert, de quatro pontos ( 0 a 3), de autorresposta. Cada subescala 
é composta por sete itens que avaliam os estados emocionais de depressão, ansiedade e estresse. Vale ressaltar que a subescala de depressão avalia sintomas como inércia, anedonia, disforia, falta de interesse, autodepreciação, desvalorização da vida e desânimo. Já a subescala de ansiedade avalia excitação do sistema nervoso autônomo, efeitos musculoesqueléticos, ansiedade situacional e experiências subjetivas de ansiedade. $E$ finalmente a subescala de estresse avalia dificuldade em relaxar, excitação nervosa, fácil perturbação/agitação, irritabilidade/ reação exagerada e impaciência ${ }^{14,15}$.

Para a realização da análise estatística dos dados, utilizou-se o software estatístico Statistical Package for the Social Sciences (SPSS) versão 20 para Windows. Após a transcrição dos dados para SPSS, realizaram-se análise exploratória para reconhecimento das variáveis e correção de eventuais erros e/ ou inconsistência da coleta e digitação dos dados.

Para a consecução do objetivo deste trabalho, a análise dos dados consistiu na descrição deles por meio de médias, desvios padrão, cálculo da consistência interna (alfa de Cronbach) ${ }^{16}$ dos instrumentos DUREL e DASS-21, análise de variância (ANOVA), teste de Welch ${ }^{17-19}$ e teste de qui-quadrado de independência ${ }^{20}$. O intervalo de confiança foi constituído com $95 \%$ de confiança, e o p-valor foi de 0,05 em todos os testes estatísticos realizados.

\section{RESULTADOS}

O grupo analisado constituiu-se de 267 acadêmicos do curso de Medicina. A idade média deles foi de 22,16 $( \pm 2,90)$, com a mínima de 18 e a máxima de 30 anos. Do total, 184 eram do sexo feminino, representando aproximadamente $68,91 \%$ da amostra, e 83 do sexo masculino, cerca de 31,09\% da amostra. Quanto à etnia declarada, 90,26\% eram brancos, 1,5\% era negro, $0,37 \%$ optou por não declarar, e os demais pertenciam a outras etnias. Por meio da Tabela 1, ainda é possível analisar a afiliação religiosa dos estudantes.

Quanto às estatísticas descritivas de frequências absoluta ( $\mathrm{n}$ ) e relativa (\%) do instrumento DUREL, obtiveram-se os resultados apresentados na Tabela 2.

Após essa primeira etapa, avaliou-se a consistência interna dos instrumentos utilizados. Para tal, lançou-se mão do coeficiente alfa de Cronbach, que é uma propriedade inerente ao padrão de resposta da população estudada. Obtiveram-se os seguintes resultados de medida de fidedignidade: 0,90 para o instrumento de DUREL e 0,93 para o questionário DASS-21. Isso demonstra que esses instrumentos oferecem medidas precisas e não apresentam dados redundantes.

Para avaliar se havia diferença nos índices de transtornos emocionais entre os acadêmicos de períodos distintos, procedeu-se à verificação de possíveis significâncias estatísticas entre os escores médios desses transtornos, realizando análise de variância (ANOVA) e também teste de Welch.

A ANOVA do escore de ansiedade dos acadêmicos em seus respectivos períodos de graduação constatou que não havia significância ( $p$-valor $=0,45$ ), ou seja, o período de graduação cursado pelo aluno não influencia no seu nível de ansiedade. Os dados coletados possibilitam ainda a análise do nível de ansiedade dos acadêmicos em seus respectivos períodos, como demonstra o Gráfico 1.

Com relação ao escore de depressão, foi necessário realizar um teste mais robusto por conta das violações de homogeneidade demonstrada pela ANOVA ( $p$-valor $=0,001$ ): o teste de Welch, que diagnostica se as médias amostrais são, ou não, estatisticamente iguais. O resultado alcançado por meio do software estatístico mostrou que os escores médios de depressão dos diferentes períodos de graduação não são estatisticamente iguais ( $p$-valor < $0,05) \mathrm{p}$-valor (teste de Welch) $=0,048$. Para verificarmos em que ponto havia essa diferença, lançamos mão do teste post hoc de least significant difference (LSD), e, por meio dele, comprovou-se que havia diferença significativa entre o escore médio de depressão dos acadêmicos do segundo período e o dos discentes do sexto período, pois $p$-valor $<0,05$ ( $p$-valor $=0,025$ ), sendo maior o escore médio de depressão dos alunos do segundo período. Também houve diferença estatisticamente significativa entre o escore médio de depressão dos acadêmicos do décimo segundo período e o do sexto período, $p$-valor $<0,05$ ( $p$-valor $=0,020$ ), sendo maior o escore médio de depressão dos alunos do décimo segundo. Por último, houve diferença estatisticamente significativa entre o escore médio de depressão dos acadêmicos do décimo segundo período e o do décimo primeiro período, $\mathrm{p}$-valor $<0,05$ ( $p$-valor $=$

Tabela 1. Afiliação religiosa dos acadêmicos.

\begin{tabular}{|c|c|c|}
\hline \multirow{2}{*}{ Afiliação religiosa } & \multicolumn{2}{|c|}{ Frequências } \\
\hline & $\mathbf{N}$ & $\%$ \\
\hline Católico & 123 & 46,07 \\
\hline Protestante & 23 & 9,74 \\
\hline Espírita & 33 & 12,36 \\
\hline Outra & 3 & 1,12 \\
\hline Não acredita em Deus & 21 & 7,87 \\
\hline Acredita em Deus, mas não tem religião & 48 & 17,98 \\
\hline Acredita em forças & 1 & 0,37 \\
\hline Adventista & 1 & 0,37 \\
\hline Budista & 1 & 0,37 \\
\hline Católico e espírita & 4 & 1,50 \\
\hline Católico, espírita e umbandista & 1 & 0,37 \\
\hline Umbandista & 1 & 0,37 \\
\hline Agnóstico & 4 & 1,50 \\
\hline
\end{tabular}

Fonte: Elaborada pelos autores. 
0,042), sendo maior nos acadêmicos do décimo segundo. Entre os demais períodos, não houve diferença.

Com base no exposto, é possível afirmar que o período de graduação cursado influenciou o nível de depressão dos acadêmicos. Ademais, o Gráfico 2 possibilita análise do nível de depressão entre os estudantes

No que tange ao estresse, verificou-se, por meio da análise do $p$-valor $>0,05$ ( $p$-valor $=0,23$ ) da ANOVA, que não havia diferença significativa entre os escores médios de estresse dos indivíduos nos diferentes períodos de graduação. Ademais, por meio do Gráfico 3, é possível observar o nível de estresse dos acadêmicos em seus respectivos períodos de graduação.

Em relação à prevalência dos transtornos mentais analisados, constatou-se que a maioria dos acadêmicos apresentava níveis normais de ansiedade, depressão e estresse, com a frequência relativa de 56,18\%, 66,29\% e $45,69 \%$, respectivamente. É válido salientar que uma parcela considerável apresentava tais transtornos de modo mais intenso, sendo $22,47 \%$ dos acadêmicos ansiosos, $21,73 \%$ estressados e $7,21 \%$ depressivos em níveis severo e extremamente severo.

Tabela 2. Estatísticas descritivas do instrumento DUREL.

\begin{tabular}{|c|c|c|c|c|c|c|c|}
\hline \multicolumn{8}{|c|}{ Perfil dos acadêmicos quanto à religiosidade organizacional (RO) } \\
\hline \multirow{3}{*}{$\begin{array}{l}\text { Frequência que vai a igreja, templo ou } \\
\text { outro encontro religioso. }\end{array}$} & & $\begin{array}{l}\text { Mais do que } \\
\text { uma vez/ } \\
\text { semana }\end{array}$ & $\begin{array}{l}\text { Uma vez/ } \\
\text { semana }\end{array}$ & $\begin{array}{l}\text { De duas a } \\
\text { três vezes/ } \\
\text { mês }\end{array}$ & $\begin{array}{l}\text { Algumas } \\
\text { vezes no ano }\end{array}$ & $\begin{array}{l}\text { Uma vez/ } \\
\text { ano ou } \\
\text { menos }\end{array}$ & Nunca \\
\hline & $\mathrm{n}$ & 14 & 40 & 28 & 88 & 49 & 48 \\
\hline & $\%$ & 5,24 & 14,98 & 10,49 & 32,96 & 18,35 & 17,98 \\
\hline \multicolumn{8}{|c|}{ Perfil dos acadêmicos quanto à religiosidade não organizacional (RNO) } \\
\hline \multirow{3}{*}{$\begin{array}{l}\text { Frequência que se dedica a atividades } \\
\text { religiosas individuais (rezas, meditações, } \\
\text { leitura de textos religiosos). }\end{array}$} & & $\begin{array}{l}\text { Mais que uma } \\
\text { vez ao dia }\end{array}$ & $\begin{array}{l}\text { Diaria- } \\
\text { mente }\end{array}$ & $\begin{array}{l}\text { Duas ou } \\
\text { mais vezes/ } \\
\text { semana }\end{array}$ & $\begin{array}{l}\text { Uma vez/ } \\
\text { semana }\end{array}$ & $\begin{array}{l}\text { Poucas } \\
\text { vezes/mês }\end{array}$ & $\begin{array}{l}\text { Raramente } \\
\text { ou nunca }\end{array}$ \\
\hline & $\mathrm{n}$ & 17 & 90 & 40 & 16 & 39 & 65 \\
\hline & $\%$ & 6,36 & 33,71 & 14,8 & 5,99 & 14,61 & 24,34 \\
\hline \multicolumn{8}{|c|}{ Perfil dos acadêmicos quanto à religiosidade intrínseca (RI) } \\
\hline \multirow{3}{*}{$\begin{array}{l}\text { Em minha vida, sinto a presença de } \\
\text { Deus ou do Espírito Santo. }\end{array}$} & & $\begin{array}{l}\text { Totalmente } \\
\text { verdade para } \\
\text { mim }\end{array}$ & $\begin{array}{l}\text { Em geral é } \\
\text { verdade }\end{array}$ & $\begin{array}{l}\text { Não estou } \\
\text { certo }\end{array}$ & $\begin{array}{l}\text { Em geral } \\
\text { não é } \\
\text { verdade }\end{array}$ & $\begin{array}{l}\text { Não é } \\
\text { verdade }\end{array}$ & \\
\hline & $\mathrm{n}$ & 128 & 67 & 27 & 21 & 24 & \\
\hline & $\%$ & 47,94 & 25,09 & 10,11 & 7,87 & 8,99 & \\
\hline \multirow{2}{*}{$\begin{array}{l}\text { As minhas crenças religiosas estão } \\
\text { realmente por trás de toda minha } \\
\text { maneira de viver. }\end{array}$} & $\mathrm{n}$ & 60 & 95 & 38 & 30 & 44 & \\
\hline & $\%$ & 22,47 & 35,58 & 14,23 & 11,24 & 16,48 & \\
\hline \multirow{2}{*}{$\begin{array}{l}\text { Eu me esforço muito para viver a religião } \\
\text { em todos os aspectos da minha vida. }\end{array}$} & $\mathrm{n}$ & 37 & 90 & 48 & 33 & 59 & \\
\hline & $\%$ & 13,96 & 33,71 & 17,97 & 12,36 & 22,1 & \\
\hline
\end{tabular}

Fonte: Elaborada pelos autores.

Gráfico 1.Nível de ansiedade e período de graduação.

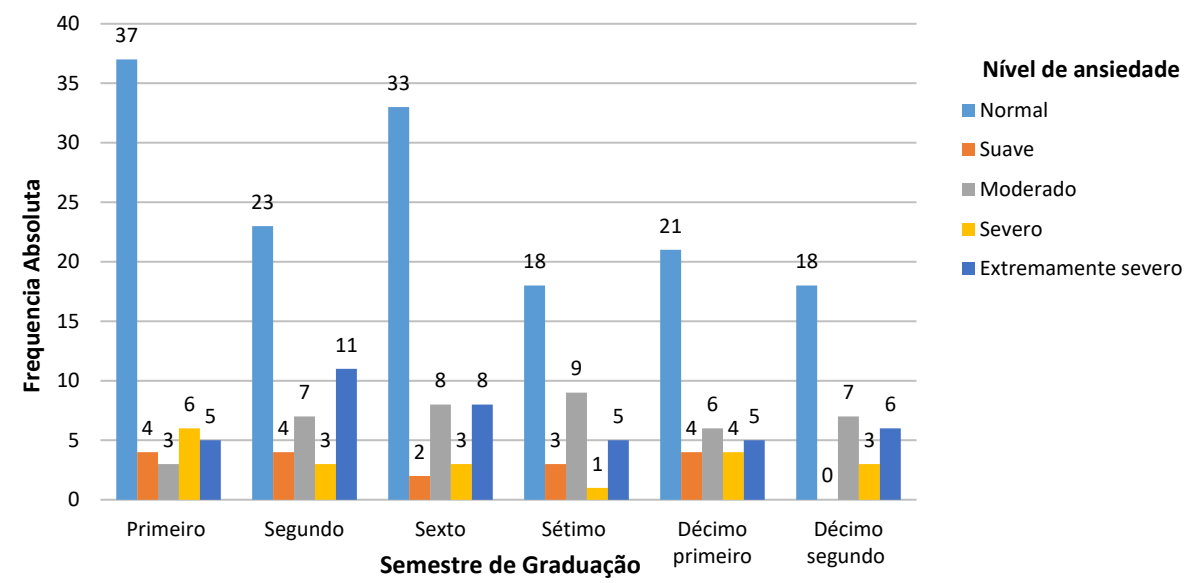

Fonte: Elaborado pelos autores. 
Gráfico 2.Nível de depressão e período de graduação

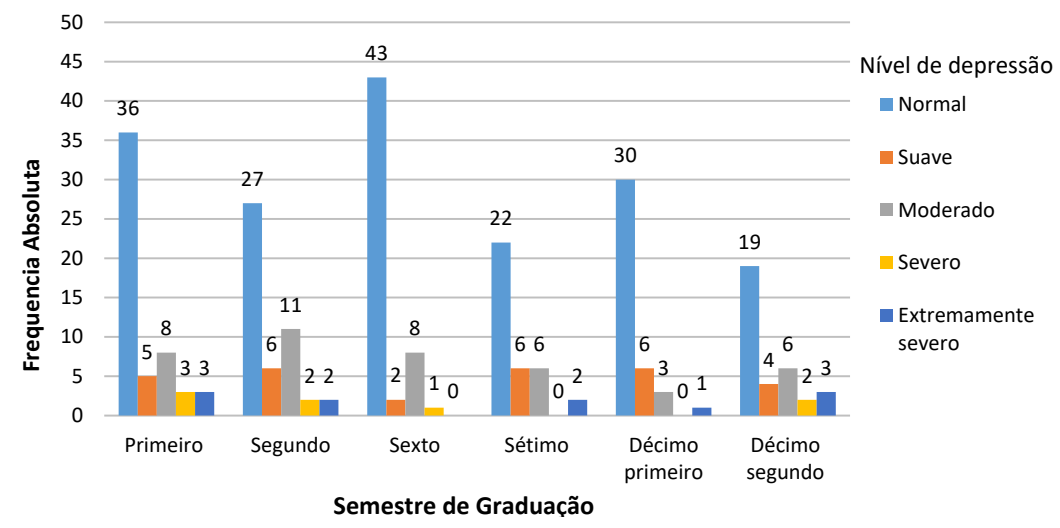

Fonte: Elaborado pelos autores.

Gráfico 3. Nível de estresse e período de graduação

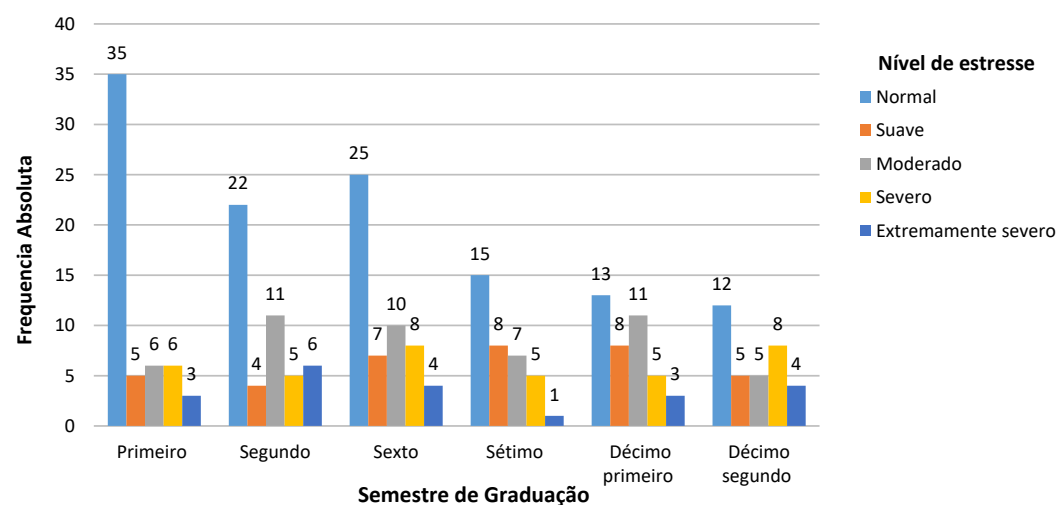

Fonte: Elaborado pelos autores.

Por fim, avaliamos a associação de aspectos religiosos e os transtornos emocionais supracitados. Como visto na Tabela 2, duas das três dimensões do Índice de Religiosidade DUREL, RO e RNO, são compostas, cada uma, por uma pergunta contendo seis alternativas, as quais especificam o nível de associação do acadêmico com aquela respectiva dimensão. A fim de possibilitar associações entre esse instrumento e a escala de transtornos emocionais, DASS-21, fizeram-se as seguintes conversões:

- $\quad$ Alternativas 1, 2 e 3 das perguntas $\rightarrow$ "Associação positiva" à religiosidade;

- Alternativas 4, 5 e 6 das perguntas $\rightarrow$ "Ausência de associação".

Já na dimensão RI, composta por três assertivas (RI 1, RI 2 e RI 3), cada uma com cinco alternativas que especificam o nível de associação com essa dimensão, a conversão foi:

- Alternativas 1 e 2 das assertivas $\rightarrow$ "Associação positiva";

- Alternativas 3, 4 e 5 das assertivas $\rightarrow$ "Ausência de associação".
- De semelhante modo, os níveis dos transtornos emocionais (normal, suave, moderado, severo e extremamente severo) foram convertidos em:

- "Ausência de sinais" do transtorno emocional observado, anteriormente chamado de nível "normal";

- "Presença de sinais" do transtorno emocional observado, anteriormente chamado de níveis "suave", "moderado", "severo" e "extremamente severo".

Por meio do teste de qui-quadrado $(x 2)$ de independência, foi examinado se a presença ou ausência de transtornos emocionais está relacionada com a associação positiva, ou negativa, a aspectos da religiosidade. Os resultados mostraram que a dimensão RO não possui associação com os transtornos emocionais depressão ( $p$-valor $=0,65$ ), ansiedade ( $p$-valor $=0,43$ ) e estresse ( $p$-valor $=0,23$ ) dos acadêmicos, e o grau de associação entre essas variáveis, externada por meio da medida Phi, foi de 3\%, 5\% e 7\%, respectivamente.

Com relação à dimensão $\mathrm{RNO}$, os resultados também revelaram que essa dimensão não possui associação com os transtornos emocionais depressão ( $p$-valor $=0,69$ ), ansiedade $(p$-valor $=0,69)$ e estresse $(p$-valor $=0,77)$ dos acadêmicos, e 
o grau de associação entre essas variáveis foi de $2 \%$, $2 \%$ e $2 \%$, respectivamente.

De modo semelhante, a dimensão RI não possui associação com os transtornos emocionais depressão ( $p$-valor $=0,83$ ), ansiedade ( $p$-valor $=0,12)$ e estresse $(p$-valor $=0,09)$ dos estudantes, e o grau de associação entre essas variáveis foi de 1\%, 9\% e 10\%, respectivamente. Com relação ao item $\mathrm{RI} 2$, também não houve associação significativa [depressão ( $p$-valor $=0,83$ ), ansiedade ( $p$-valor $=0,20$ ) e estresse ( $p$-valor $=0,23$ ), e o grau de associação entre essas variáveis foi de $12 \%, 8 \%$ e $7 \%$, respectivamente]. Por último, os resultados do item RI 3 foram: $p$-valor $=0,21$ para sinais de depressão; ( $p$-valor $=0,93$ ) para sinais de ansiedade; e ( $p$-valor $=0,63$ ) para sinais de estresse, e o grau de associação entre essas variáveis foi de $8 \%, 1 \%$ e $3 \%$, respectivamente.

\section{DISCUSSÃO}

Dados apresentados pelo último censo do Instituto Brasileiro de Geografia e Estatística (IBGE) ${ }^{21}$ demonstram que a população brasileira é, majoritariamente, cristã, de afiliação católica, mas há um aumento no número de pessoas não afiliadas a nenhuma religião, o que está bem representado na amostra do presente estudo.

A despeito de os valores religiosos ainda serem muito utilizados entre jovens com alto nível de escolaridade, população cotidianamente exposta a situações que afetam sua saúde mental ${ }^{20}$, foi demonstrado que todas as dimensões de RO, RNO e RI não possuem associação estatisticamente significativa com os transtornos emocionais depressão, ansiedade e estresse dos acadêmicos. Isso pode ser fundamentado no fato de a R/E apresentarem caráter qualitativo, difícil de ser mensurado ${ }^{10} \mathrm{com}$ a utilização de instrumentos universais, mesmo que validados, sendo necessário apurar as individualidades da amostra.

Numa comparação com artigos científicos previamente publicados, em relação à depressão, nosso estudo corrobora o resultado obtido por Gonçalves et al. ${ }^{22}$ : nenhuma das variáveis de $R / E$ estava associada à depressão em uma amostra de estudantes da área da saúde (Medicina e Enfermagem). Além disso, esse mesmo estudo concluiu que a RO foi um fator protetor para ansiedade, mas esse resultado divergente pode ser justificado por diferenças metodológicas do estudo, já que os autores utilizaram uma escala de autoespiritualidade e a escala hospitalar de ansiedade e depressão.

Um estudo semelhante com estudantes de Israel $^{23}$ também não apresentou associação entre $R / E$, depressão e ansiedade, mostrando que os resultados podem ser semelhantes mesmo em culturas distintas.

Gonçalves et al. ${ }^{6}$, em metanálise, revelaram que, apesar das correlações positivas, existem também associações entre
R/E e pensamentos pessimistas de culpa ou punição, como cometer um pecado, que culminam em resultados negativos maior prevalência de depressão e ansiedade. O poder da crença nesses pensamentos de perda pode ser influenciado, entre outras variáveis, pelo índice de religiosidade e, dessa forma, estar concatenado a maior índice de depressão 9 .

Nossos resultados ainda reforçam as teses ${ }^{24,25}$ de que os desafios do princípio e do final da faculdade ${ }^{14}$ - experiência do desconhecido e responsabilidades - exacerbam a angústia dos acadêmicos. Desse modo, a prevalência de transtornos mentais nos acadêmicos de Medicina é alta, principalmente nos períodos extremos da graduação (segundo e décimo segundo).

Por sua vez, Bassols et al. ${ }^{26}$, em um estudo brasileiro, encontraram maior prevalência de sintomas de ansiedade em estudantes de Medicina do primeiro ano quando comparados àqueles do fim da graduação, o que destoa dos resultados obtidos com o presente estudo, visto que apenas os sintomas depressivos obtiveram escores médios, significativamente diferentes, entre os períodos.

Em relação às limitações, a não associação entre R/E e saúde mental encontrada reforça a necessidade da realização de estudos longitudinais para investigar os aspectos multidimensionais mais prevalentes nas diferentes religiões.

Por ser uma pesquisa transversal, o momento da aplicação dos questionários pode constituir um viés, visto que os estressores não são homogêneos ao longo do semestre, como o período de retorno após as férias e proximidade das avaliações. Como o estudo foi realizado com acadêmicos de uma única instituição, a generalização exige cautela, já que a exigência acadêmica e o apoio psicossocial podem interferir nos resultados ${ }^{22}$. Outro aspecto referente aos artigos é a dificuldade de definir conceitos complexos e multifacetados como R/E, pois não há uma definição universal'.

Entretanto, o presente estudo também apresenta consideráveis pontos fortes. Os questionários utilizados são validados no idioma dos voluntários, de fácil aplicação e compreensão, possuindo reprodutibilidade mundial ${ }^{14}$.

\section{CONCLUSÃO}

Considerando o exposto, é possível propor recomendações para pesquisas futuras, de modo que os resultados possam ser utilizados em estudos de metanálise, com investigação de aspectos multidimensionais mais prevalentes em diferentes religiões, sendo necessário, ainda, realizar mais pesquisas longitudinais. Por fim, é importante ressaltar que os estudos, como este, que não apresentaram relação significativa entre as variáveis não podem ser negligenciados para que sejam investigadas as dimensões positivas e negativas da associação entre R/E e saúde mental. 


\section{CONTRIBUIÇÃO DAS AUTORAS}

Larissa Cruvinel Leite e Larissa Vitoria Dornelas elaboraram o projeto, coletaram os dados, discutiram os achados e redigiram o artigo. Laura de Souza Bechara Secchin orientou o projeto e a execução do trabalho, e contribuiu para a revisão do texto.

\section{CONFLITO DE INTERESSES}

As autoras declaram não haver conflito de interesses neste estudo.

\section{FINANCIAMENTO}

Declaramos que não houve financiamento para a realização desta pesquisa.

\section{REFERENCES}

1. Wong YJ, Rew L, Slaikeu KD. A systematic review of recent research on adolescent religiosity/spirituality and mental health. Issues Ment Health Nurs. 2006;27(2):161-83.

2. Kioulos KT, Bergiannaki JD, Glaros A, Vassiliadou M, Alexandri Z, Papadimitriou G. Religiosity dimensions and subjective health status in Greek students. Psychiatriki. 2015;26(1):38-44.

3. Carlotto RC. Spirituality and depressive symptoms in Brazilian college students. Rev Psicol UNESP. 2013;12(2):50-60.

4. Zanetti GC, Lemos GL, Gotti ES, Tomé JM, Silva AP, de Rezende EAMR. The perception of medical students as well as students from other healthrelated areas regarding the relations between spirituality, religiosity and health. Rev Bras Educ Med. 2018;42(1):65-72.

5. Koenig HG, McCullough M, Larson DB. Handbook of religion and health: a century of research reviewed. 2nd ed. New York: Oxford University Press; 2001.

6. Gonçalves JPB, Lucchetti G, Menezes PR, Vallada H. Religious and spiritual interventions in mental health care: a systematic review and meta-analysis of randomized controlled clinical trials. Psychol Med. 2015;45(14):2937-49.

7. Koenig HG, Pearce MJ, Nelson B, Daher N. Effects of religious versus standard cognitive-behavioral therapy on optimism in persons with major depression and chronic medical illness. Depress Anxiety. 2015;32(11):835-42.

8. Pikó B, Kovács E. Is religiosity a protective factor? Social epidemiologic study of adolescent psychological health. Orv Hetil. 2009;150(41):1903-8.

9. Vasegh S, Mohammadi MR. Religiosity, anxiety, and depression among a sample of Iranian medical students. Int J Psychiatry Med. 2007;37(2):213-27.

10. Chaves EC, lunes HD, Moura CC, Carvalho CL, Silva AM, Carvalho EC. Anxiety and spirituality in university students: a cross-sectional study. Rev Bras Enferm. 2015;68(3):504-9.

11. Chaves, F. Religião, religiosidade ou espiritualidade? A importância da definição dos termos no contexto de saúde física e psíquica. POHSA. 2015;5(14).
12. Taunay TCE, Gondim FAA, Macêdo DS, Moreira-Almeida A, Gurgel LA, Andrade LMS, et al. Validity of the Brazilian version of the Duke Religious Index (DUREL). Rev Psiquiatr Clín. 2012;39(4):130-5.

13. Koenig HG, Büssing A. The Duke University Religion Index (DUREL) a five-item measure for use in epidemiological studies. Religions. 2010;1(1):78-85.

14. Moutinho IL, Maddalena NC, Ronald RK, Lucchetti ALG, Tibiriça SHC, Ezequiel OS, et al. Depression, stress and anxiety in medical students: a cross-sectional comparison between students from different semesters. Rev Assoc Med Bras. 2017;63(1):21-8.

15. Apóstolo JLA, Mendes AC, Azeredo ZA. Adaptation to Portuguese of the Depression, Anxiety and Stress Scales (DASS). Rev Lat Am Enfermagem. 2006;14(6):863-71.

16. Almeida D, Santos MAR, Costa AFB. Aplicação do coeficiente alfa de Cronbach nos resultados de um questionário para avaliação de desempenho da saúde pública. Anais do XXX Encontro Nacional de Engenharia de Produção. Maturidade e desafios da engenharia de produção: competitividade das empresas, condições de trabalho, meio ambiente; 12-15 out. 2010; São Carlos, SP, Brasil. São Carlos: Abepro; 2010

17. Blanca MJ, Alarcón R, Arnau J. Non-normal Data: is ANOVA still a valid option? Pshicothema. 2017;29(4):552-7.

18. Montgomery DC. Design and analysis of experiments. 9th ed. Hoboken, NJ: John Wiley \& Sons; 2017.

19. Scheffé H. The analysis of variance. New York: John Wiley \& Sons; 1999.

20. Campbell I. Chi-squared and Fisher-Irwin tests of two-by-two tables with small sample recommendations. Stat Med. 2007;26(19):3661-75.

21. Instituto Brasileiro de Geografia e Estatística. Censo Demográfico 2010 Características gerais da população, religião e pessoas com deficiência. Rio de Janeiro: IBGE; 2010 [access in 24 jul 2020]. Available from: http:// biblioteca.ibge.gov.br/visualizacao/periodicos/94/cd_2010_religiao_ deficiencia.pdf.

22. Gonçalves JRL, Jorge AP, Zanetti GC, Amaro EA, Tótoli RT, Lucchetti G. Religiousness is associated with lower levels of anxiety, but not depression, in medical and nursing students. Rev Assoc Med Bras. 2018;64(6):537-42.

23. Lupo MK, Strous RD. Religiosidade, ansiedade e depressão entre estudantes de medicina israelenses. Isr Med Assoc J. 2011;13(10):613-8.

24. Miller P, Surtees P. Sintomas psicológicos e seu curso em estudantes de medicina do primeiro ano, avaliados pelo Interval General Health Questionnaire (I-GHQ). Ir J Psiquiatria. 1991;159(2):199-207.

25. Quince TA, Wood DF, Parker RA, Benson J. Prevalence and persistence of depression among undergraduate medical studentes: a longitudinal study at one UK medical school. BMJ Open. 2012;2(4):e001519.

26. Bassols AM, Okabayashi LS, Silva AB, Carneiro BB, Feijó F, Guimarães GC, et al. First- and last-year medical students: is there a difference in the prevalence and intensity of anxiety and depressive symptoms. Rev Bras Psiquiatr. 2014;36(3):233-40. 